\title{
Engineering Ethics: A Critical Dimension of the Profession
}

\author{
doi:10.3991/ijep.v1i2.1639 \\ Nael Barakat \\ Grand Valley State University, Grand Rapids, MI, USA
}

\begin{abstract}
Engineering has always had a massive impact on human health and welfare. Unfortunately, the public only realizes the magnitude of this impact when very few engineering disasters occur, like huge oil spells in the sea or the failure of an aero-plane or a building. This is in spite of the plethora of engineering systems working perfectly around the clock to enhance every miniature aspect of public health and welfare.
\end{abstract}

The ethical dimension of the engineering profession deals with the interaction with the public. However, engineering ethics are critical for reasons beyond keeping out of legal trouble and guarding the health and safety of humans. Ethics are necessary for the survival and continuity of the profession itself, amongst other reasons. Therefore, engineering codes of ethics have been set by professional societies and engineering ethics have been emphasized by accreditation organizations to be an integral part of the engineering curricula. In addition, ethics is the framework that allows the handling of evolving issues related to the profession of engineering. Examples of these issues include the globalization of the practice, continuous professional development (CPD) of the practitioners, and issues of emerging technologies (e.g. nanotechnology).

In the midst of this huge dimension, the engineering instructor is challenged to incorporate engineering ethics in a packed curriculum. This paper will provide a quick overview of the basic concepts and definitions of engineering ethics as well as the importance of studying engineering ethics. Some recent engineering ethics challenges will be listed with focus on globalization, its influence on the professional and ethical side of the profession, and the perspective of the educator. Moreover, some suggested strategies and best practices to integrate engineering ethics in the curriculum will be discussed.

Index Terms - Engineering Ethics, professional engineering, Engineering ethics education, workforce development.

\section{INTRODUCTION}

On January 28, 1986 the Challenger spaceship exploded just over a minute into its flight. Seven astronauts were killed while being watched over global media by millions of viewers. The failure was blamed mostly on engineering technical errors which involved ethical considerations [1].

Similar cases of ethical misconduct involving engineers have taken the front media stage like the Kansas City Hyatt-Regency Hotel walkway collapse [1], and the BP oil spell in the Gulf of Mexico [2]. In spite of the media daily flood of ethical misconduct stories involving politicians, entertainers, and others, ethical misconduct by professionals like engineers and medical doctors always takes differ- ent dimensions. One reason is that ethical misconduct by professionals, relating to their profession, will most likely inflect harm and cause physical damages to multiple human lives, property, or both. This is one small, but most widely felt, part of the societal impact by engineering endeavors. In fact, the accumulation of these experiences is a major reason that drives the public to be very cautious regarding new and untested technology. This is evident in the case of nanotechnology, where the media frequently presents articles and analyses about this promising technology that cover a mixture of facts and speculations, ranging from unrealistic high expectations, through realistic information, and to unfounded and exaggerated fears [3].

It is evident that the rapid pace of technological advances will continue to confront engineers with ethical questions that have never existed. These questions will be posed by concerned members of the society. Nevertheless, these members are usually not the experts in the technical sides of issues and consequently their questions will bring about many levels of thinking which can easily lead to unpredictable interpretations and misinformation of the public. In many cases, the public has direct influence on the continuity and success of these technological endeavors, which makes it imperative that engineers and scientists fulfill their double duties, as citizens with special expertise, and correctly bring out the accurate information for the public to make informed decisions about any questions surrounding a technological endeavor or product [4].

That brings us to the basic concept of engineering as a social contract. The public grants engineers the rights to self regulate and practice their profession in exchange for the great benefits they bring to society while safeguarding and protecting the health and welfare of that society. Therefore, it is critical that engineering professionals consider and integrate the societal impact of their professional practice. This aspect of the profession falls under what is known as engineering ethics. Most professional organizations have produced codes of ethics to help their members practice safer engineering and to help the public see the commitment of the profession to public safety and welfare.

The Accreditation Board for Engineering and Technology (ABET) has also focused attention, in its criteria for accrediting engineering programs, on this side as a critical part of the set of soft skills necessary to provide engineering students with the needed awareness of the societal impact of their professional practice [5]. Governmental funding agencies, like the National Science Foundation (NSF) of the USA, are now strictly requesting ethics training of personnel operating on project funded by the agency and are requesting the integration of ethical dimensions 
in proposals for funding engineering and scientific projects, based on their mandates from the government [6].

This paper will provide an overview of engineering ethics and societal impact and how it is emphasized by professional engineering organizations through instruments like the codes of ethics. The importance of integrating this part into engineering curricula will also be discussed. Following, will be a review of examples of a contemporary engineering ethics challenges, like globalization. Moreover, some suggested strategies and a listing of some best practices to integrate engineering ethics in engineering curricula by educators will be presented.

\section{BASIC DEFINITION OF ENGINEERING ETHICS}

Common knowledge about ethics tends to mix engineering ethics with religion, etiquette, or common morality. It is true that many basic philosophies are shared between all of these systems and engineering ethics. However, engineering ethics is definitely none of the above. Engineering ethics is a wide frame work that brings most of the non-technical aspects of the profession, including the professional, human, and societal ones, into engineering practice. In addition, engineering ethics provide a forum for discussing and integrating evolving issues in engineering.

Ethics, or morality, are defined as the standards of proper conduct. In spite of some dictionary differences, the words "ethics" and "morality" are usually used interchangeably in the current context. Ethical philosophies originate from culture norms filtered through religious traditions among other filters. That is another reason for applied ethics being always confused with religion, etiquette, law, and other social norms. One of the relevant definitions to be mentioned here is common morality, which is the set of moral ideals shared by most members of a culture or society.

Another source of confusion between professional ethics and other ethical platforms is that a boundary between personal and professional ethics is not always clear. Personal ethics are the set of one's own ethical commitments, acquired during upbringing and later modified. How a person deals with and treats others on a day-to-day basis is based mostly on personal ethics. However, professional ethics are the set of standards of conduct adopted by professionals. This is different than the laws of the profession which include the set of laws governing the practice of the profession and enforced by public law enforcement and licensing agencies. Nevertheless, most of the laws of the profession are ethically based. However, ethical does not always mean legal and vice versa. Consider the following example: "A hypothetical regulation by the Environmental Protection Agency (EPA) states that no more than 10 PPM of a particular pollutant should exist in the water output from a factory into a lake. That makes any concentration above 10 PPM illegal. However, is it ethical to dispose of water when the pollutant concentration is 7 PPM? It is clearly legal, but is it ethical?"

This is the type of dilemma for which a good background and knowledge base in professional engineering ethics is needed. Once an engineer is to make a decision relevant to the previous example, the factors to be considered would include ethics and societal impact as well as legal and economical issues. However, ethical considera-

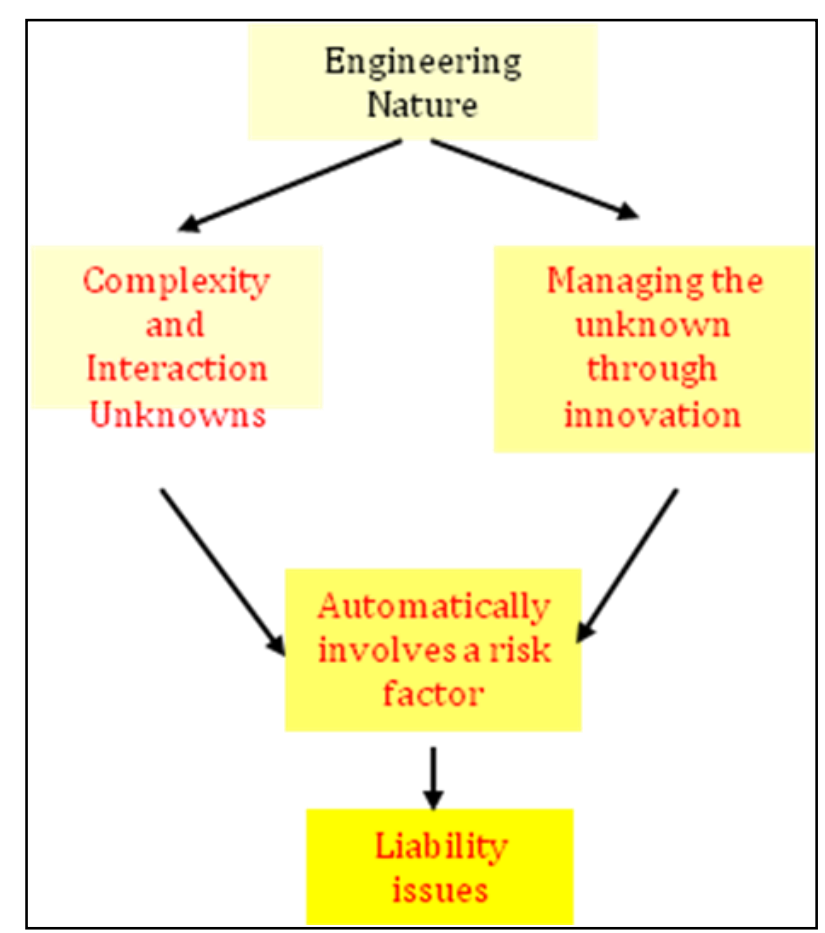

Figure 1. Liabilities associated with the nature of engineering.

tions are always above and beyond legal and political correctness.

The definition and function of engineering ethics requires an initial understanding of professional ethics followed by a critical determination of whether engineering is indeed a profession. Once that is determined, engineering ethics, its function, and many other relevant concepts related to the nontechnical side of the engineering profession will become evident and almost intuitive to understand.

\section{THE NATURE OF ENGINEERING}

The nature of engineering (fig. 1) requires managing the unknown and involves interactions amongst many unknowns with innovative complex systems. This nature automatically involves higher risk factors and, in turn, many liability issues, especially if the final outcome is to deal with humans, resources, or the environment. Because of this very nature, engineering projects are considered social experiments requiring morally responsible engineering behavior. Engineers have a responsibility for protecting the safety and well-being of the public according to what some might consider "an implicit social contract." At this point, you might wonder about this responsibility and contract and where it came from. This is directly related to the true nature and definition of a profession, which, in turn, leads to the more fundamental questions of whether engineering is a profession or not. To answer this question, an examination of the attributes of a profession would significantly help.

\section{DEFINITION OF A Profession}

A profession is historically known as a free act of commitment to a way of life. The dictionary defines a profession as the occupation which one professes to be skilled in and follow [7]. In fact, there is no universal definition for a profession, and henceforth the identifica- 
tion of the attributes of a profession would be more helpful.

The attributes of a profession can be divided into the following three categories:

A. Membership requirements:

1) Extensive formal education and training of intellectual character.

2) Sophisticated skills, autonomy, and use of judgment; not routine.

B. Public and society relations:

1) Vitally need for the knowledge and skills of the members for society well-being.

2) Hence ... professional organizations are allowed by the public to self control/set/enforce the practice.

\section{Professional relations:}

Professionals are normally regulated by ethical

standards, embodied in a Code of Ethics.

A close examination of these attributes against engineering would result in a positive identification of engineering as a profession. Indeed engineering products and services do influence the public health and welfare to a great extent. The fact that most people are not aware of this, compared to the influence of a profession like medical doctors (MDs) is characterized by the answer to the following question: How many times did you get to meet your MD as opposed to the engineer who designed your car or manufactured it? The answer would definitely be an eye opener to the reason of why the public is not fully aware of what engineers do.

\section{NEED TO STUDY ENGINEERING ETHICS}

Based on the previously established concepts of professional ethics, engineering being a profession, and henceforth engineering ethics need and function, studying engineering ethics can be referred to as the study of moral and societal impact issues and decisions confronting individuals and organizations engaged in engineering. It is also the study of questions related to the moral conduct, ideals, character, policies, and relationships of people and corporations involved in technological activities. In summary, engineering ethics should be studied and understood by engineers because of the following reasons:

A. Engineering by nature is a profession of managing the unknown, and that involves higher risks, which reflect on the end users (the public).

B. To sensitize engineers to important ethical issues before they have to confront them and to create appropriate awareness that can influence the earliest stages of engineering endeavors.

C. To develop "moral autonomy" and enable engineers to self regulate and guard the safety and welfare of the public as well as the profession, regardless of the environment and location of operation.

D. To train engineers to analyze complex problems and resolve them in the most ethical manner, without compromising their personal ethics or their professional obligations.

One last concept to establish here is that engineering ethics is not about doing the right thing when the ethical choice is obvious. It is actually about finding the middle ground in a complex ethical dilemma that will achieve most benefits and least damages to all parties, without compromising the basics. These basics are: public safety and resources, the client, the engineer, and the profession.

\section{CODES OF ETHICS}

Codes of ethics are simply defined as the shared standards of professional ethics. These codes help establish common standards of professional conduct and a two way agreement; 1) amongst the professionals themselves, providing a rationale for the professionals to adhere to, and 2) between the professionals and the public, to promote public well-being, gain public trust, and stay competent. In addition, codes of ethics provide a focus for debate on evolving issues including professional engineering ethics.

There are codes of ethics for almost every engineering organization, whether it is comprehensive, like the National Society of Professional Engineers (NSPE) [8] or by discipline, like the American Society of Mechanical Engineers (ASME) [9] or The Institute of Electrical and Electronic Engineers (IEEE) [10]. However, all codes of engineering ethics have the following common attributes:

A. A primary obligation to protect the safety and respect the right of consent of human subjects, as well as conserve natural resources and reduce damage to the environment.

B. A constant awareness of the experimental nature of any project, imaginative forecasting of its possible side effects, and a reasonable effort to monitor them.

C. A personal involvement in all steps of a project and an acceptance of accountability for the results of a project.

D. An obligation to protect and advance the reputation and integrity of the profession and the professionals.

Codes of ethics have become an essential part of the training provided to engineering students in most ABET accredited engineering programs. ABET requires ethics education as part of its accreditation criteria of engineering programs [5]. Nevertheless, education of engineers regarding the ethical and societal impact of their activities is critical for many reasons beyond the fulfillment of ABET requirements or being legally safe. Codes of ethics can be an excellent tool for effective handling of ethical dilemmas by engineers and engineering students as well as for effective teaching and training of engineers on how to integrate ethics into their professional activities.

When sorting out an ethical dilemma, one usually refers to the relevant codes of ethics depending on the nature of the dilemma. For example, if the dilemma is related to common or personal issues, one usually relies on own beliefs, values gained during one's upbringing, sense of decency, or common sense. In professional dilemmas, guidelines by the relevant professional organization, characterized in its code of ethics should be the first reference to sort out that a dilemma. Nevertheless, it should be emphasized here that codes of ethics are only a framework for ethical judgment and critical analysis for a professional. A code of ethics is not a recipe or a substitute for sound judgment. Moreover, a code of ethics is neither a legal document nor a source of moral or ethical principles. 


\section{CONTEMPORARY ETHICAL ISSUE}

Engineering ethics and related codes are the medium that allows for discussing and integrating evolving issues in the profession. Two examples of these issues will be described next. These include the issue of globalization of engineering practice and the issue of continuous professional development of engineers after joining the workforce.

Globalization of engineering practice and education continues to be a challenge in all aspects. Globalization has become so prominent in engineering education and activities. This is an issue that is critical to engineering but is not only technical in nature. Fortunately, engineering ethics is the appropriate home to handle this issue and the like. From an educator's point of view, an up to date curriculum must include the international perspective to continue producing competent engineers, capable of functioning in different societies and cultures. This component can be handled within the engineering ethics education on how to practice engineering within constraints by the social and political system which are completely different from what is known to the engineer, without jeopardizing the engineering activity or compromising the ethical standards of the profession. Early exposure to such possible situations during the educational period of the engineer's preparation is not only necessary but also provides them with an edge in the marketplace [11]. Figure 2 illustrates the issues and interactions related to the globalization of engineering.

Continuous professional development (CPD) is also an issue that is difficult to manage by engineers in the workforce. Educating engineers at an early stage on how to continue learning and improve their skills and knowledge has become a critical necessity dictated by competence requirements, as well as by engineering ethics and professionalism. Today, CPD is important to practice safe and useful engineering and to maintain licensure. Integrating CPD into the professional practice and optimizing its parts and choices to achieve maximum benefit for the engineer is a challenging task. Engineering ethics is the home to handle such important, but non-technical side of engineering. This is yet another side of ethics that is not directly detected from the title [12].

\section{INTEGRATING ENGINEERING ETHICS IN THE CURRICULUM}

As was previously mentioned, it is no surprise that engineering ethics is now a required component in engineering education and continuous professional development. To educate engineers and engineering students about ethics, different strategies can be implemented as appropriate to the curriculum or context at hand. Meanwhile, a couple of challenges for the engineering educators continue to persist when approaching the topic of teaching engineering ethics. These challenges in a simplified form are: the lack of expertise amongst the engineering faculty to teach such a non-technical topic, and the lack of room in a typical engineering curriculum to accommodate this additional material. Nevertheless, engineers are in the business of problem solving and the literature has multiple reports on methods to overcome these challenges. Following is a list of strategic elements to consider when teaching engineering ethics:

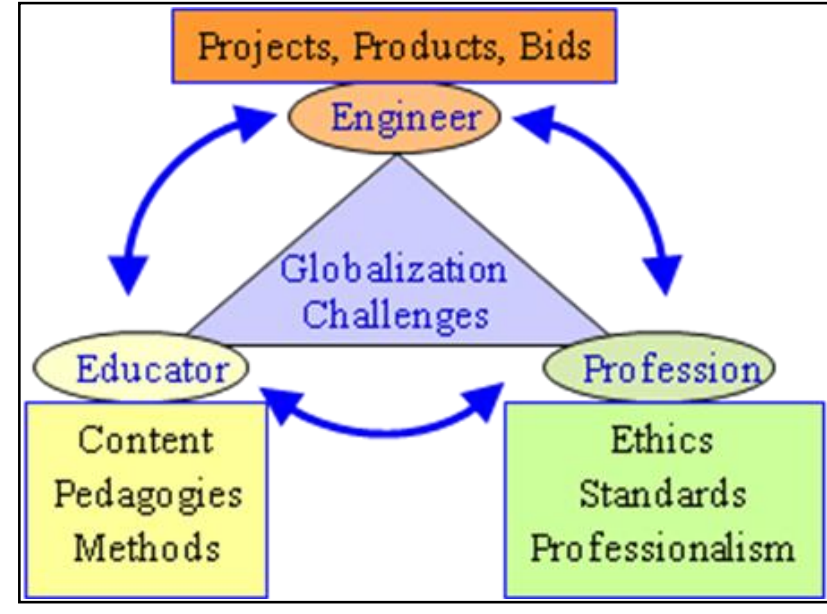

Figure 2. Globalization of engineering issues and interaction.

A. Engineering students should learn engineering ethics similar to the way they learn technical topics. As they build awareness of the ethical issues related to engineering and as they learn the tools available for handling ethical dilemmas, like the codes of ethics, they should be able to extrapolate this knowledge and anticipate ethical traps before they occur. That knowledge should also be applicable to new situations and scenarios.

B. Ethical considerations and societal impact should be integrated into engineering activities at the very early stages. As an example, ethical and environmental impacts can be included as design constraints or functional requirements in a product or process. This way the ethical part will effectively influence all decisions appropriately instead of being an ad hoc activity or an after-the-fact batch that has no effective influence on any part of the activity. As students become trained on this concept and applying it, the idea of including the ethical dimension becomes part of the practice that does not require any reminders.

C. While considering the ethical aspects in an engineering activity, a balance is required to maintain progress in the activity with caution and attention to its ethical constraints. Ethical considerations should be carefully stated and built on solid scientific and factual bases, not on speculative or unfounded arguments.

D. Engineers should be very strongly encouraged to take an active part in social and political discussions related to engineering as part of the ethics training to fulfill their double duty as citizens and as experts in the field. This will guarantee the safeguarding of the reputation and integrity of the profession as well as the continuity of public trust resulting in the continued independence of the profession.

E. Engineering educators should capitalize on engineers' inherited training and their ability to detect patterns and comfortably deal with numbers and formulae. Ethical dilemma resolution using simulation to a design problem and the projection of the relevant parts of the code of ethics on the problem at hand helps tremendously in training the engineer on 


\section{SHORT PAPER \\ ENGINEERING ETHICS:A CRITICAL DIMENSION OF THE PROFESSION}

understanding and utilizing the codes of ethics in actual and practical situations.

F. A strategy that is more pertinent to laboratory work and research is to continuously attach an ethics tag question to every step in a design, build, or experimental step. By asking at every step along the way about the effect of that step on safety, human life, and other relevant societal issues (e.g. environment, sustainability, etc!), the practice becomes an integral built-in part of every engineering activity. A follow up set of questions should request steps to mitigate risks if they exist and steps to balance risk vs. benefit.

From a content point of view, the education on engineering ethics should include the following elements:

A. Ethics basics and concepts, which are covered in the earlier part of this paper. As a minimum, knowledge of codes of ethics and why they exist as well as how to use them should be covered.

B. Examples and cases studies involving ethical dilemmas faced by engineers during practice. These are available in the literature and any engineering ethics text book [1].

C. Expansion above and beyond legal and minimum requirements for an ethical practice to reach the concepts of good-works.

D. Extrapolation of engineering ethics to cover contemporary issues like globalization.

E. Applications in actual processes and products like decisions in material selection and resources' preservation.

Engineering ethics can be integrated in the curriculum in many formats. These include full courses or modules added to technical courses. When using modules, it is preferred that a thread be built in the curriculum that capitalizes on previous knowledge and uses the context of the course for applying ethical concepts taught within that course. Some schools would introduce their own honor code as well, starting at the freshman year, to familiarize the student with it and introduce the professional side of engineering at the school level [13].

\section{CONCLUSION}

Engineering ethics is an integral part of the profession that provides a frame work to handle most non-technical and emerging issues, as well as societal impact and interaction with the surroundings. However, it is definitely not to be mixed with religion, etiquette, or other common morality concepts. Engineering ethics is a critical part of engineering education to produce competent engineers. This is a challenging part for the engineering educator that requires some planning to be integrated in the curriculum. Strategies exist to incorporate engineering ethics in the curriculum including separate courses or modules sprinkled in the curriculum to form a thread. To achieve an effective engineering ethics education, engineers must understand the dimensions and impact of the profession, the need for ethics to protect the public, the engineer and the profession, and the need for integrating ethics in engineering endeavors early and effectively. Early exposure to actual and practical situations to apply engineering ethics and solve possible ethical dilemmas properly equips engineers to deal with such scenarios in a correct and lawful manner, regardless of the environment and location of operation.

\section{REFERENCES}

[1] C. Harris, M. Pritchard, and M. Rabins, "Engineering Ethics, Concepts and Cases," $4^{\text {th }}$ edition, 2009, Wadseorth Cengage Learning, Belmont, Ca.

[2] Robertson, Campbell; Krauss, Clifford (2010-08-02). "Gulf Spill Is the Largest of Its Kind, Scientists Say". The New York Times (The New York Times Company). http://www.nytimes.com/ 2010/08/03/us/03spill.html? r=1\&fta=y. Retrieved 2010-11-23.

[3] Nael Barakat, "The Practical Scope of Nanotechnology Ethics," Environmental Nano Technologies (ENT) Magazine, issue 3, IANET Association, Rome, Italy, on line: www.entmagazine.com, to appear 2011.

[4] Barakat N. and H. Jiao, "Proposed Strategies for Teaching ethics of Nanotechnology," Nanoethics Journal, Springer, Netherlands, Sep. 2010. DOI 10.1007/s11569-010-0100-0.

[5] 2010-2011 Criteria for Accrediting Engineering Programs, Engineering Accreditation Commission of ABET, ABET Inc. Baltimore, MD. 2009, www.ABET.org, Retrieved Nov. 2010.

[6] America COMPETES Act, Public Law 110-69-Aug 9, 2007, section 7009, the Library of Congress (THOMAS) online, http://thomas.loc.gov/cgi-bin/bdquery/z?d110:s.00761: retrieved Nov 25, 2010

[7] Merriam-Webster dictionary online, www.merriam-webster.com, retrieved Nov. 25, 2010

[8] National Society of Professional Engineers (NSPE), "NSPE Code of Ethics for Engineers," http://www.nspe.org/Ethics/ CodeofEthics/index.html, accessed Nov. 2010.

[9] American Society of Mechanical Engineers (ASME - International), "ASME code of ethics," http://www.asme.org/Education/ PreCollege/TeacherResources/Code Ethics_Engineers.cfm, (Accessed Nov. 2010).

[10] Institute of Electrical and Electronic Engineers (IEEE), "IEEE Code of Ethics," http://www.ieee.org/membership_services/ membership/ethics_code.html, accessed Nov. 2010.

[11] Barakat N. "Upgrading Engineering Graduate for a World-Class Practice," ASEE NCS, March 2007, Charleston, WV

[12] Barakat N., "Merging Continuous Professional Development into Engineering Education and Practice," ASEE-NCS Annual Conference, Grand Rapids, MI. 2009

[13] Fleischmann S. "Essential Ethics-Embedding Ethics into an Engineering Curriculum," Journal of Science and Engineering Ethics, Vol. 10, P. 369-381, April 2004, doi:10.1007/s11948-0040033-5.

\section{AUTHOR}

Nael Barakat, Ph.D. P.Eng. FASME* is Mechanical Engineering Program Chair at School of Engineering, Grand Valley State University, 301 W Fulton St. Grand Rapids, MI. USA - 49504, barakatn@gvsu.edu

*Member and chair elect (2011-2014) of the Committee on Ethical Standards and Review (CESR) of the American Society of Mechanical Engineers (ASME-International).

Received April 20 $0^{\text {th }}, 2011$. Published as resubmitted by the author June $6^{\text {th }}, 2011$. 